\title{
Protein interactions with HER-family receptors can have different characteristics depending on the hosting cell line
}

\author{
PAVEL BARTA $^{1 *}$, JENNIE MALMBERG $^{2 *}$, LUDMILA MELICHAROVA $^{1}$, JOHN STRANDGÅRD $^{3}$, \\ ANNA ORLOVA $^{2}$, VLADIMIR TOLMACHEV ${ }^{4}$, MILAN LAZNICEK ${ }^{1}$ and KARL ANDERSSON ${ }^{3,4}$ \\ ${ }^{1}$ Department of Pharmacology and Toxicology, Faculty of Pharmacy in Hradec Kralove, Charles University in Prague, \\ Heyrovského 1203, 50005 Hradec Králové, Czech Republic; ${ }^{2}$ Preclinical PET Platform, Uppsala University, \\ Dag Hammarskjölds väg 14C, SE 75183 Uppsala; ${ }^{3}$ Ridgeview Instruments AB, Ulleråkersv 62, \\ SE 75643 Uppsala; ${ }^{4}$ Department of Radiology, Oncology, and Radiation Sciences, \\ Rudbeck Laboratory, Uppsala University, SE 75185 Uppsala, Sweden
}

Received October 14, 2011; Accepted November 29, 2011

DOI: 10.3892/ijo.2011.1307

\begin{abstract}
Cell lines are common model systems in the development of therapeutic proteins and in the research on cellular functions and dysfunctions. In this field, the protein interaction assay is a frequently used tool for assessing the adequacy of a protein for diagnostic and therapeutic purposes. In this study, we investigated the extent to which the interaction characteristics depend on the choice of cell line for HER-family receptors. The interaction characteristics of two therapeutic antibodies (trastuzumab and cetuximab) and one Affibody molecule $\left(\mathrm{Z}_{\mathrm{HER} 2: 342}\right)$, interacting with the intended receptor were characterized with high precision using an automated realtime interaction method, in different cell lines $(\mathrm{HaCaT}$, A431, HEP-G2, SKOV3, PC3, DU-145). Clear differences in binding affinity and kinetics, up to one order of magnitude, were found for the interaction of the same protein binding to the same receptor on different cells for all three proteins. For HER-family receptors, it is therefore important to refer to the measured affinity for a protein-receptor interaction together with the hosting cell line. The ability to accurately measure affinity and kinetics of a protein-receptor interaction on cell lines of different origins may increase the understanding of underlying receptor biology, and impact the selection of candidates in the development of therapeutic or diagnostic agents.
\end{abstract}

Correspondence to: Dr Karl Andersson, Ridgeview Instruments AB, Ulleråkersv 62, SE 75643 Uppsala, Sweden

E-mail: karl@ridgeviewinstruments.com

*Contributed equally

Key words: HER2, epidermal growth factor receptor, affinity, trastuzumab, cetuximab, affibody molecules

\section{Introduction}

Cell-based assays are commonly used in the development of therapeutic proteins and in the research on the underlying biology of cellular functions and dysfunctions. As the smallest element of living matter, cloned cells in culture represents an in vitro model system that reflects essential molecular function of tissues in vivo, such as signaling, metabolism and cell membrane transport. Protein interaction assays are one subclass of cell-based assays and they are particularly common in the development of diagnostic and therapeutic proteins, such as therapeutic antibodies or molecular imaging agents.

One important question is the validity of any particular cell line as a model for the disease under study. This question is very important in oncology, where the tumor cell is by definition altered into an immortal state. There is increasing evidence that the choice of model cell line can impact the estimation of the efficacy (1), or the pharmacokinetics (2), or the apparent affinity (3-5) of a compound by a factor 3-10. The validity of the cell line as a model has long-lasting impact on the development of therapeutic proteins and fundamental research, because it questions the scope of reported findings: Is the observed effect general for the disease under study or is the effect limited to the currently used model system?

The cell-based assays for protein-cell interactions have essentially remained the same during the last 40 years: Most assays rely on incubation of labeled protein with the cell culture, followed by a wash and finally a quantification of the amount of retained label on the cells after a wash. Even though the read-out modalities have been improved with increased sensitivity and throughput, the basic assay principle of incubate-wash-quantify has remained the same. Recent development in protein design and affinity maturation has however increased the binding strength of the proteins to $\mathrm{K}_{\mathrm{D}}<1 \mathrm{nM}$, which leads to inherent problems with the assay principle of incubate-wash-quantify since the time required to reach equilibrium often exceeds $10 \mathrm{~h}$ (6). Therefore, we apply a novel type of assay capable of measuring how proteins interact with molecular targets on living cells in real-time, LigandTracer ${ }^{\circledR}(7)$. This assay is conducted 
on an inclined, slowly rotating petri dish and was applied on protein-receptor interactions on different hosting cell lines.

This study is focused on interaction of targeting proteins with receptors belonging to HER (human epidermal growth factor receptor) tyrosine kinases family. Signaling and crossinteraction of HER family is described in a number of reviews (8-11). The HER family consists of four homologous receptors: EGFR (epidermal growth factor receptor, also called HER1), HER2, HER3 and HER4, and ten known ligands. One commonly accepted mechanistic model is that binding of a ligand to a HER receptor causes profound conformational changes, leading to homo- and heterodimerisation, even though other theories have been presented (12). Of note, HER2 has no known ligand, but is capable of dimerizing with other members of HER family without ligand binding (11). Cellular responses to HER receptor tyrosine kinase activation are numerous, and include cell division, differentiation and motility, as well as apoptosis suppression.

It is well documented that excessive HER signaling, arising from receptor overexpression, mutations or autocrine stimulation, is a hallmark of a wide variety of solid tumors $(8,10)$. Currently, several monoclonal antibodies targeting different receptors of HER 2 family are approved for clinical use and a number are under active development (13).

Our main hypothesis is that for targeting proteins binding to HER-family receptors, the interaction characteristics will be strongly dependent on the choice of cell line. To verify this hypothesis, we performed a detailed characterization of three different molecular interactions: ${ }^{131}$ I-cetuximab - EGFR, ${ }^{125}$ I-trastuzumab - HER2 and Affibody molecule ${ }^{111}$ In-Z342 HER2, each conducted on three different cell lines.

\section{Materials and methods}

Cell lines. The cell lines used in this study were human squamous carcinoma cell line A431 (Health Protection Agency, Salisbury, UK), the human ovarian carcinoma cell line SKOV3 (HTB-77, ATCC, Rocksville, MD, USA), the human prostate cancer bone metastasis cell line PC3 (CRL-1435, ATCC), the human prostate cancer brain metastasis cell line DU-145 (HTB-81, ATCC), the human keratinocyte cell line HaCaT (DKFZ, Heidelberg, Germany), and the human Caucasian hepatocyte carcinoma cell line HEP-G2 (Health Protection Agency). The cells were seeded on a small local area of a petri dish (Nuclon $^{\mathrm{TM}}$, dish size 100x20, NUNC A/S, Roskilde, Denmark) as previously described (7). Cells were cultivated in RPMI cell culture medium (Biochrom AG, Berlin, Germany) for SKOV3, PC3 and DU-145 cells, Eagle's MEM cell culture medium (Sigma-Aldrich, Germany) for A431 and Hep-G2 cells and Dulbecco's MEM cell culture medium (Sigma-Aldrich) for HaCaT cells. Cell culture media were supplemented with $10 \%$ fetal calf serum (FCS, Sigma, St. Louis, MO, USA), L-glutamin (2 mM, Biochrom AG), PEST (penicillin $100 \mathrm{IU} / \mathrm{ml}$ and streptomycin $100 \mu \mathrm{g} / \mathrm{ml}$ for SKOV3, PC3 and DU-145 only, Biochrom AG). The cells were cultivated at $37^{\circ} \mathrm{C}$ in incubator with humidified atmosphere and $5 \% \mathrm{CO}_{2}$ until experimental day.

Radiolabeling. Monoclonal antibody cetuximab $(80 \mu \mathrm{g})$ (purified from Erbitux, Merck KGaA, Darmstadt, Germany) were labeled with $10 \mathrm{MBq}{ }^{131} \mathrm{I}$ (Institute of Isotopes Co., Ltd. Budapest, Hungary) using Chloramine-T according to the protocol (14). Monoclonal antibody trastuzumab (80 $\mu \mathrm{g})$ (purified from Herceptin, Roche AB, Stockholm, Sweden) was labeled with 5-10 MBq ${ }^{125}$ I (Perkin-Elmer, Wellesley, MA, USA) using the same protocol (14). The labeling reactions were performed with chloramine-T (Sigma) and sodium metabisulfite (Aldrich, Stockholm, Sweden). The desired radiolabeled protein was purified on a NAP-5 column (GE Healthcare, Waukesha, WI, USA) equilibrated with PBS (10 mM, pH 7.4, $140 \mathrm{mM} \mathrm{NaCl}$ ). Affibody molecule $\mathrm{Z}_{\text {HER2:342 }}$ was pre-conjugated with DOTA $(1,4,7,10$-tetraazacyclododecane-1,4,7-tris-acetic acid-10-maleimidoethylacetamide) and radiolabeling with ${ }^{111}$ In (Covidien, Hazelwood, MO) through chelation was performed essentially as previously described (15).

Real-time interaction measurements in LigandTracer. Real-time measurements of the binding of labeled proteins to their respective receptors on the cells were performed at room temperature in LigandTracer ${ }^{\circledR}$ (Ridgeview Instruments AB, Uppsala, Sweden). LigandTracer Yellow was used for ${ }^{131}$ I-cetuximab and Affibody molecule ${ }^{111}$ In-Z342 measurements, and LigandTracer Grey for ${ }^{125} \mathrm{I}$-trastuzumab measurements, all according to previously published protocol (3). After a short baseline measurement, the cells were incubated first at a lower concentration of protein, then at a higher concentration and finally the cells were put in fresh cell culture medium to monitor the release of bound material. The concentrations and incubation times used for the different proteins are shown in Table I. All measurements were repeated at least twice. Affinity estimations were made using non-linear fits to the 'OneToOne', 'OneToOneDepletionCorrected' or the 'OneToTwo' interaction model in TraceDrawer 1.3 (Ridgeview Instruments $\mathrm{AB})$.

Interaction Map analysis. The mathematical method Interaction Map $(12,16)$ expresses the measured binding of a (homogeneous) ligand to a heterogeneous group of targets as a sum of interactions, each having a unique combination of the association rate constant $\mathrm{k}_{\mathrm{a}}$ and dissociation rate constant $\mathrm{k}_{\mathrm{d}}$ :

MeasuredCurve $=\sum_{i=1}^{n} \sum_{j=1}^{m}\left[W_{i j} \mathrm{x}\right.$ CurveComponent $\left(\right.$ conc $\left.\left., k_{a}^{i}, k_{d}^{j}\right)\right]$

where conc is the concentration of protein in solution (the 'ligand') and $W_{i j}$ is the weighing factor describing the contribution to the measured real-time interaction curve. The calculated contributing curves are represented as grayscale (black $=$ large $W_{i j}$, white $=$ small $W_{i j}$ ) peaks in an on-off plot. In this study, the Interaction Map method used $24\left(k_{a}\right)$ x $30\left(k_{d}\right)$ different nodes with kinetic parameter values evenly distributed in $\log$-space $\left(\log 10\left(\mathrm{k}_{\mathrm{a}}\right)=\{2.00,2.25,2.50, \ldots, 7.25,7.50\right.$, $\left.7.75\}, \log 10\left(\mathrm{k}_{\mathrm{d}}\right)=\{-6.60,-6.40,-6.20, \ldots,-1.20,-1.00,-0.80\}\right) . \mathrm{A}$ Tichonov-type regularization algorithm was employed, adding penalty to the sum-of-square residuals if there are many peaks in the Interaction Map. A similar algorithm has been presented previously (17) and has been applied to SPR-based real-time interaction analysis. 
Table I. Concentrations and incubation times used in LigandTracer assays.

\begin{tabular}{lccccc}
\hline Protein & Label & First incubation & Second incubation & Third incubation & Retention measurement \\
\hline Cetuximab & ${ }^{131} \mathrm{I}$ & $3 \mathrm{nM}, 4-5 \mathrm{~h}$ & $15 \mathrm{nM}, 5-6 \mathrm{~h}$ & - & $0 \mathrm{nM}, \sim 5 \mathrm{~h}$ \\
Trastuzumab & ${ }^{125} \mathrm{I}$ & $1 \mathrm{nM}, 2-3 \mathrm{~h}$ & $4 \mathrm{nM}, 2-3 \mathrm{~h}$ & $7 \mathrm{nM}, 2-3 \mathrm{~h}^{\mathrm{a}}$ & $0 \mathrm{nM}, \sim 5 \mathrm{~h}$ \\
$\mathrm{Z}_{\mathrm{HER} 2: 342}$ & ${ }^{111} \mathrm{In}$ & $1 \mathrm{nM}, 2-3 \mathrm{~h}$ & $4 \mathrm{nM}, 2-3 \mathrm{~h}$ & - & $0 \mathrm{nM}, \sim 5 \mathrm{~h}$ \\
\hline
\end{tabular}

In most assays, the binding protein was incubated at two different concentrations. ${ }^{\mathrm{a} F o r}{ }^{125} \mathrm{I}$-trastuzumab measurements on DU-145 and PC 3 cells, a third concentration was applied as well.

Table II. Affinity values for the investigated protein-receptor interactions.

\begin{tabular}{|c|c|c|c|c|c|c|}
\hline Targeting protein & Molecular target & Cell line & $\mathrm{K}_{\mathrm{D} 1}(\mathrm{pM})$ & Prevalence $(\%)$ & $\mathrm{K}_{\mathrm{D} 2}(\mathrm{nM})$ & Prevalence $(\%)$ \\
\hline${ }^{131}$ I-cetuximab & EGFR & A 431 & $135 \pm 40$ & 100 & - & \\
\hline${ }^{131}$ I-cetuximab & EGFR & $\mathrm{HaCaT}$ & $119 \pm 21$ & 100 & - & \\
\hline${ }^{131}$ I-cetuximab & EGFR & HEP-G2 & $\approx 10$ & 100 & - & \\
\hline${ }^{125} \mathrm{I}$-trastuzumab & HER2 & SKOV3 & $100 \pm 50$ & 100 & - & \\
\hline${ }^{125}$ I-trastuzumab & HER2 & PC3 & $90 \pm 50$ & $\sim 10$ & $80 \pm 11$ & $\sim 90$ \\
\hline${ }^{125}$ I-trastuzumab & HER2 & DU-145 & $120 \pm 100$ & $\sim 50$ & $29 \pm 17$ & $\sim 50$ \\
\hline${ }^{111} \mathrm{In}-\mathrm{Z}_{\mathrm{HER} 2: 342}$ & HER2 & SKOV3 & $50 \pm 30$ & 100 & & \\
\hline${ }^{111}$ In- $Z_{\text {HER2:342 }}$ & HER2 & PC3 & $13 \pm 4$ & $\sim 15$ & $10^{-8}$ & $\sim 85$ \\
\hline${ }^{111}$ In- $Z_{\text {HER2:342 }}$ & HER 2 & DU-145 & $46 \pm 5$ & $\sim 40$ & $10^{-8}$ & $\sim 60$ \\
\hline
\end{tabular}

In cases where two affinities were derived for an interaction, the estimated relative prevalence is reported.

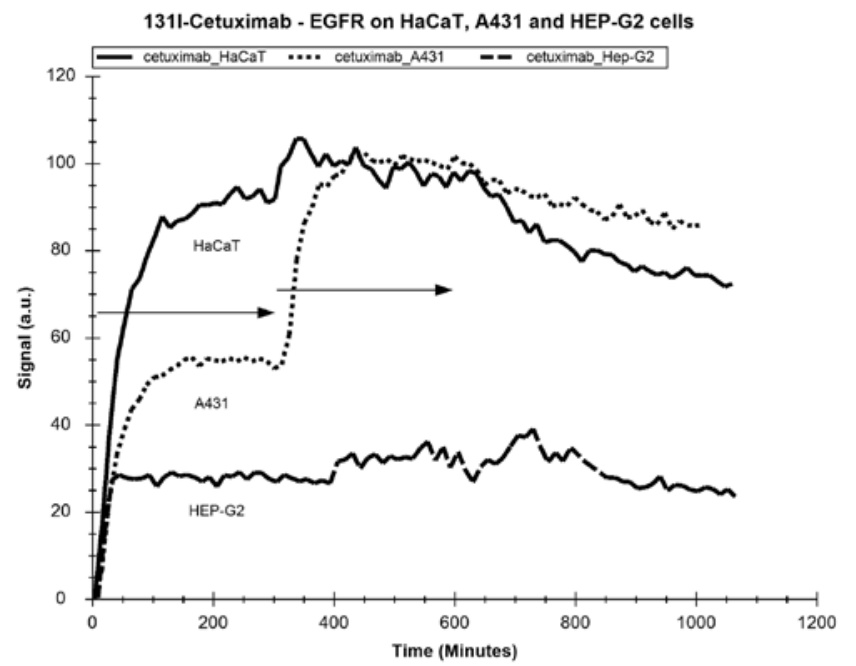

Figure 1. Binding curves representing ${ }^{131}$ I-cetuximab interacting with EGFR on three different cell lines. ${ }^{131}$ I-cetuximab was used at two different concentrations (indicated with two arrows); $3 \mathrm{nM}$ for the initial 4-5 h, followed by $15 \mathrm{nM}$ for another 5-6 h, followed by a wash-out measurement in pure cell culture medium (i.e. $0 \mathrm{nM}{ }^{131} \mathrm{I}$-cetuximab). The signal levels from $\mathrm{HaCaT}$ and A431 were comparable, and HEP-G2 measurements resulted in significantly lower signal level. Curves have been scaled to enhance visibility.

\section{Results}

${ }^{131}$ I-cetuximab - EGFR. Binding curves from ${ }^{131}$ I-cetuximab interacting with A431, HaCaT and HEP-G2 cell lines are shown in Fig. 1. There were clear differences in how the ${ }^{131}$ I-cetuximab binding progress in time. The results for the cell lines A431 and $\mathrm{HaCaT}$ may look different, but this difference is related to assay conditions. The large number of EGFR receptors per A431 cell (2x10\% cell) (18) depletes the antibody-containing medium of unbound antibody, resulting in lower effective antibody concentration at equilibrium during the incubation of the lower concentration. Thus an interaction model that accounts for depletion of antibody is required for accurate analysis of data for A431. The interaction to HEP-G2 is very rapid (i.e. short time to reach equilibrium) and shows minor increase in signal when the concentration is elevated during the second incubation step. The HEP-G2 binding curves do not contain sufficient curvature for a detailed kinetics and affinity analysis. The estimated affinities for the interactions are provided in Table II.

${ }^{125}$ I-trastuzumab-HER2 . Binding curves from ${ }^{125}$ I-trastuzumab interacting with HER2 on SKOV3, PC3, and DU-145 cell lines are shown in Fig. 2. The binding of ${ }^{125}$ I-trastuzumab to HER2 on SKOV3 is slower than to HER 2 on the other cell lines. When comparing signal magnitude, SKOV 3 cells have approximately 2 orders of magnitude more HER 2 receptors per cell than the others. The binding profiles of the three interactions are very different: ${ }^{125} \mathrm{I}$-trastuzumab interacting with SKOV3 produced a monophasic binding profile, while as ${ }^{125}$ I-trastuzumab interacting with HER2 on PC3 cells produced a biphasic profile. For DU-145, a weak biphasic profile was found. Therefore, SKOV3 results were analyzed using the OneToOne model 


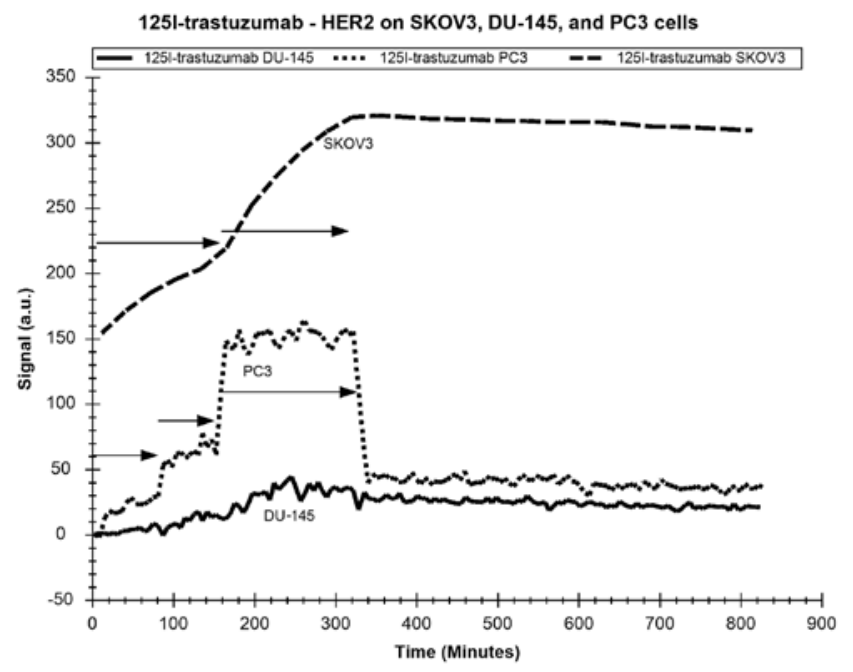

Figure 2. Binding curves representing ${ }^{125}$ I-trastuzumab interacting with HER2 on three different cell lines. ${ }^{125}$ I-trastuzumab was used at two different concentrations on SKOV3 (indicated with two arrows); first $1 \mathrm{nM}$ for the initial $3 \mathrm{~h}$, followed by $4 \mathrm{nM}$ for another 3-4 h, followed by a washout measurement in pure cell culture medium. For PC3 and DU-145, three concentrations were used (indicated with three arrows); first two shorter incubations at $1 \mathrm{nM}$ and $4 \mathrm{nM}$, followed by a $3 \mathrm{~h}$ incubation at $7 \mathrm{nM}$. Since the signal level from SKOV3 was 30-100 times higher than the signal levels from PC3 and DU-145, the curves have been scaled and moved to enhance visibility.

and the other two cell lines were analyzed using the biphasic OneToTwo model. The affinity values are presented in Table II. The binding properties of the single interaction to SKOV3 and the stronger interactions of PC3 and DU-145 are all similar.

${ }^{11} I n-D O T A-Z_{\text {HER2:342 }}-H E R 2$. Binding curves from Affibody molecule ${ }^{111}$ In- DOTA-Z $Z_{\text {HER 2:342 }}$ interacting with HER2 on PC3, DU-145, and SKOV3 cell lines are shown in Fig. 3. There are clear differences in how the ${ }^{111} \mathrm{In}-\mathrm{DOTA}-\mathrm{Z}_{\mathrm{HER} 2: 342}$ binding progress in time. The binding curves on PC 3 and DU-145 are biphasic. The association rate of ${ }^{111} \mathrm{In}-\mathrm{DOTA}-\mathrm{Z}_{\mathrm{HER} 2: 342}$ to PC 3 is higher than that to DU-145, but the affinity is approximately the same. For SKOV-3, the association rate is $\sim 10$ times slower as compared to PC3 and DU-145, and only one interaction seems to occur. The large number of HER2 receptors per SKOV-3 cell causes depletion (as described for the cetuximab measurements above) and thus a depletion-corrected analysis model was used. All estimated affinities are shown in Table II. The weaker interaction of PC3 and DU-145 was difficult to quantify, indicating that it is a weak interaction available but it is difficult to accurately estimate its properties.

Also in this case, the affinity of the ${ }^{111}$ In-DOTA- $Z_{\text {HER2:342 }}$ interaction with HER2 on SKOV-3 was close to the higher affinity of binding to PC 3 and DU-145 cells. Of note, for both prostate cancer cell lines, the approximate prevalence of binding sites with lower affinity for ${ }^{111}$ In-DOTA-Z $Z_{\text {HER2:342 }}$ was similar to the prevalence for ${ }^{125}$ I-trastuzumab.

To further illustrate the biphasic behavior of ${ }^{111} \mathrm{In}-\mathrm{Z} 342$ interacting with HER2 on PC3 and DU-145, Interaction Maps were calculated (Fig. 4). Both interactions result in Maps with two distinct peaks, one representing a high-affinity event (the leftmost peaks A1 and B1) and another representing a weaker

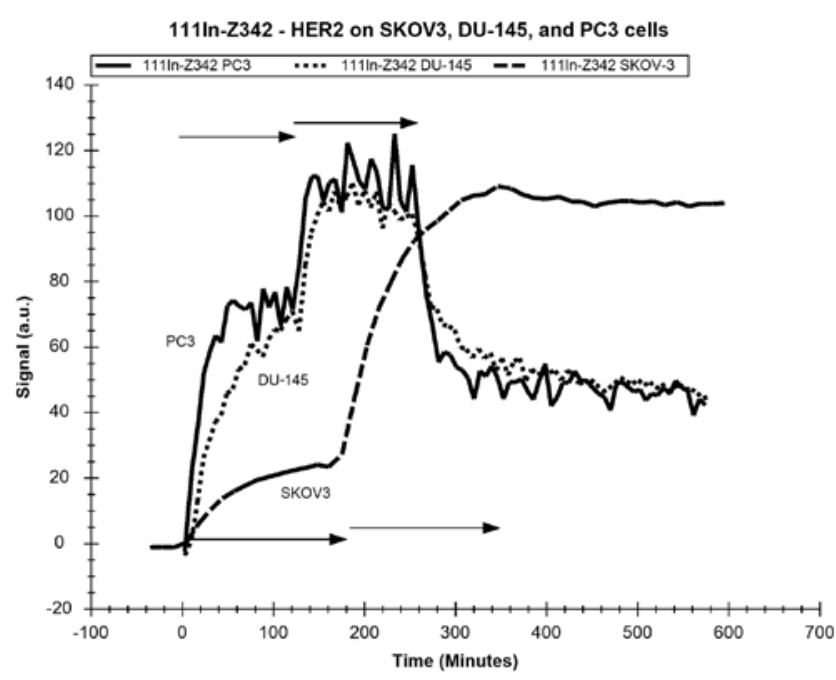

Figure 3. Binding curves representing ${ }^{111} \mathrm{In}-\mathrm{Z}_{\mathrm{HER} 2: 342}$ interacting with HER2 on three different cell lines. ${ }^{111} \mathrm{In}-\mathrm{Z}_{\mathrm{HER} 2: 342}$ was used at two different concentrations (indicated with two arrows); first $1 \mathrm{nM}$ for the initial 2-3 h, followed by $4 \mathrm{nM}$ for another 2-3 h, followed by a wash-out measurement in pure cell culture medium. For SKOV3 cells, longer incubation times were required as indicated with a separate set of arrows. The signal level from SKOV3 was $\sim 10$ times higher than the signal levels from PC3 and DU-145. The curves have been scaled to enhance visibility.

event (A2, B2). The affinities corresponding to the peaks are A1: $45 \mathrm{pM}, \mathrm{A} 2: 3.2 \mathrm{nM}, \mathrm{B} 1: 10 \mathrm{pM}, \mathrm{B} 2: 3.1 \mathrm{nM}$.

\section{Discussion}

This study confirms the hypothesis that the binding strength (affinity) and binding kinetics of targeting proteins to a cell-surface HER-family receptor can be strongly dependent on the hosting cell line. This finding is corroborated by the observation by Björkelund et al (3) that binding of a natural ligand, EGR, is different to different EGFR-expressing cell lines. Furthermore, our findings are in agreement with data (obtained by classical Scatchard analysis) that affinities of HER2- and EGFR- targeting antibodies is dependent on the cell line $(4,5)$. Thus, we issue a warning for transferring an affinity value measured on one cell line to other cell lines. We suggest that an affinity, which has been determined in a cell-based assay, should always be referred to together with the identity of the hosting cell line; otherwise the results may be misinterpreted and used in situations where they are not valid. The risk of the transferred affinity value being about one order of magnitude different is large, clearly for HER family receptors and potentially also for other receptor families.

To be more specific, for ${ }^{131}$ I-cetuximab interacting with EGFR, there is a factor 10 in affinity difference across three cell lines. For ${ }^{125}$ I-trastuzumab interacting with HER2, all three cell lines result in a strong interaction of approximately the same affinity $(\sim 100 \mathrm{pM})$, but two prostate cancer cell lines have clear biphasic behavior which is missing in the ovarian cancer cell line. Similarly, binding of ${ }^{111} \mathrm{In}$ - DOTA- $\mathrm{Z}_{\mathrm{HER} 2: 342}$ to HER2 shows clear difference in terms of interaction kinetics and in the degree of biphasic behavior. In any of these three cases, reporting the interaction characteristics from one of the 

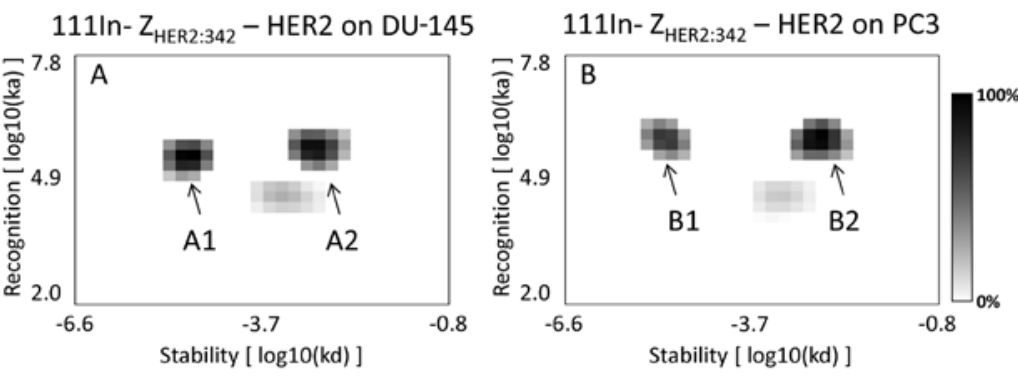

Figure 4. Interaction Maps illustrating the heterogeneity of the interactions of ${ }^{111} \mathrm{In}-\mathrm{Z}_{\mathrm{HER} 2: 342}$ - HER2 on the cell lines DU-145 and PC3. For DU-145, two different interactions are visible, one stronger (A1) with an apparent affinity of $45 \mathrm{pM}$ and one weaker (A2) with an apparent affinity of $3.2 \mathrm{nM}$. The two interactions A1 and A2 have a similar contribution to the total binding. For PC3, two interactions are visible, one stronger (B1) with an apparent affinity of 10 pM and one weaker (B2) with an apparent affinity of $3.1 \mathrm{nM}$. The weaker interaction B2 contributes more to the total interaction than the stronger interaction.

cell lines would typically not be representative for the other cell lines.

One potential origin of the observed differences is that the protein-receptor interactions on cells may be heterogeneous in a cell-dependent manner. Heterogeneity can have many underlying causes: The receptor on the cell may exist with different glycosylation patterns or as different isoforms, they may dimerize with themselves or with other receptors, or may be mutated to mention only a few possibilities. It is therefore understandable that the protein-receptor interaction in a cellular context may be very complex. This is illustrated in the Interaction Maps (Fig. 4) where ${ }^{111}$ In- DOTA-Z ${ }_{\text {HER2:342 }}$ binding to HER2 on DU-145 and PC3 are depicted as two different events of very different affinities. What these two peaks represent is not known. It is previously shown that the EGF-EGFR interaction (12) is strongly dependent on the balance between receptor dimers and monomers, and this is a potential explanation also in this case.

The observed differences in binding profile open up for speculation on the biological origin of the heterogeneity. For

${ }^{131}$ I-cetuximab-EGFR, the deviating cell line HEP-G2 is derived from liver and may have not only EGFR but also other less specific receptors for the capture and subsequent catabolism of circulating proteins. The observed interaction of ${ }^{131} \mathrm{I}$-cetuximab with EGFR on HEP-G2 is however very strong, which is an argument against less specific receptors being part of the picture. In the case of HER2 expressing cells, there is a clear difference between the prostate cancer cell lines and SKOV3, both in terms of binding characteristics, and of the relative response to the two different proteins used in this study. As assessed using ${ }^{125}$ I-trastuzumab, PC3 and DU-145 have a small amount of HER2 receptors with different binding characteristics compared to SKOV3. However, the binding of ${ }^{111} \mathrm{In}-\mathrm{DOTA}-\mathrm{Z}_{\mathrm{HER} 2: 342}$ to HER2 does not result in such large differences of receptor quantity, as evident from smaller difference in signal level across the three cell lines. PC3 and DU-145 both have signs of heterogeneity (similar for trastuzumab and $\mathrm{Z}_{\mathrm{HER} 2: 342}$ ), which is not seen on SKOV3. The origin of the differences is currently unknown, but trastuzumab and $\mathrm{Z}_{\mathrm{HER} 2: 342}$ are known to bind different receptor epitopes meaning that the Z342 epitope is more abundantly exposed. It is further known that SKOV3 expresses one order of magnitude more HER2 receptors than EGF receptors (18), while as PC3 and DU-145 have similar expression levels of the two receptors $(15,19)$. Formation of HER2-EGFR dimers is a probable factor influencing the observed heterogeneity in this case.

In the development of therapeutic or diagnostic agents, protein interaction assays provide important decisive information during the initial phases. During protein selection in selection or screening processes, candidate proteins are chosen on their ability to bind the receptor in a variety of biophysical assays. These assays may produce perfectly accurate results for the environmental conditions they represent, but do the values reflect the interaction properties in the target environment, i.e. the living cell? Since this study indicates that interaction properties can vary one order of magnitude between different hosting cell lines, it would be advisable not to discard any strong binders when moving from biophysical assays to cellbased assays. It may be that strongest binders in the biophysical assays turn out to perform moderately on cells, and that the best binder in cells is found among those performing good, but not the best, in biophysical assays. We strongly believe that a poor binder in biophysical assays will also be poor in cell-based assays.

It is well known that there is a large variation in the efficacy of therapeutic and diagnostic agents in a population of patients. The ultimate goal would be personalized medicine, wherein the patient is first characterized with respect to probable response to a variety of treatments, and then provided the treatment most likely to be effective. Understanding the underlying cause of differences in the interaction of a therapeutic or diagnostic agent with the target protein in different cell lines is one of the first important steps. This study shows that the detection technology of today can accurately map the variation across cell lines in a far more detailed manner than manual incubate-wash-quantify assays, and potentially pave the way for more detailed characterization of patients prior to selecting treatment.

In conclusion, we confirm that the binding affinity of the same protein binding to HER-family receptors can vary one order of magnitude due to cellular context. Care is advised in transferring an affinity value measured on one cell line to other cell lines, and we suggest that an affinity should always be referred to together with the identity of the hosting cell line.

\section{Acknowledgements}

The authors thank Affibody AB, Stockholm, for providing ABY-025, and Apoteket Farmaci AB (Cytostatikaberedningen, 
Sjukhusapoteket, Uppsala) for assistance in obtaining trastuzumab. The study was supported by the Grant Agency of the Czech Republic - grant No. P304/10/1738. We thank Dr Magnus Malmqvist for valuable comments on the manuscript. Ridgeview Instruments $\mathrm{AB}$ develops and manufactures the device LigandTracer. K.A. and J.S. are employed by Ridgeview Instruments $\mathrm{AB}$, and K.A. is a shareholder of Ridgeview Instruments $\mathrm{AB}$. The other authors have no conflict of interest.

\section{References}

1. Chinn DC, Holland WS, Yoon JM, Zwerdling T and Mack PC: Anti-tumor activity of the HSP90 inhibitor SNX-2112 in pediatric cancer cell-lines. Pediatr Blood Cancer: July 27, 2011 (Epub ahead of print).

2. Milenic DE, Wong KJ, Baidoo KE, Ray GL, Garmestani K, Williams $M$ and Brechbiel MW: Cetuximab: Cetuximab: preclinical evaluation of a monoclonal antibody targeting EGFR for radioimmunodiagnostic and radioimmunotherapeutic applications. Cancer Biother Radiopharm 23: 619-631, 2008.

3. Björkelund H, Gedda L and Andersson K: Comparing the epidermal growth factor interaction with four different cell lines: intriguing effects imply strong dependency of cellular context. PLoS One 6: e16536, 2011.

4. Nestor M: Effect of cetuximab treatment in squamous cell carcinomas. Tumor Biol 31: 141-147, 2010.

5. Xu FJ, Yu YH, Bae DS, Zhao XG, Slade SK, Boyer CM, Bast RC Jr and Zalutsky MR: Radioiodinated antibody targeting of the HER-2/neu oncoprotein. Nucl Med Biol 24: 451-459, 1997.

6. Andersson K, Björkelund $\mathrm{H}$ and Malmqvist M: Antibodyantigen interactions: What is the required time to equilibrium? Available from Nature Precedings <http://hdl.handle.net/10101/ npre.2010.5218.1>, 2010.

7. Björke $\mathrm{H}$ and Andersson $\mathrm{K}$ : Automated, high-resolution cellular retention and uptake studies in vitro. Appl Radiat Isot 64: 901-905, 2006.
8. Hynes NE and Lane HA: ERBB receptors and cancer: the complexity of targeted inhibitors. Nat Rev Cancer 5: 341-354, 2005.

9. Yarden Y and Sliwkowski MX: Untangling the ErbB signalling network. Nat Rev Mol Cell Biol 2: 127-137, 2001

10. Marmor MD, Skaria KB and Yarden Y: Signal transduction and oncogenesis by ErbB/HER receptors. Int J Radiat Oncol Biol Phys 58: 903-913, 2004.

11. Citri A and Yarden Y: EGF-ERBB signalling: towards the systems level. Nat Rev Mol Cell Biol 7: 505-516, 2006.

12. Björkelund H, Gedda L, Barta P, Malmqvist M and Andersson K: Gefitinib induces epidermal growth factor receptor dimers which alters the interaction characteristics with ${ }^{125}$ I-EGF. PLoS One 6: e24739, 2011.

13. Press MF and Lenz HJ: EGFR, HER2 and VEGF pathways: validated targets for cancer treatment. Drugs 67: 2045-2075, 2007.

14. Greenwood FC, Hunter WM and Glover JS: The preparation of ${ }^{131}$ I-labelled human growth hormone of high specific radioactivity. Biochem J 89: 114-123, 1963.

15. Malmberg J, Tolmachev V and Orlova A: Imaging agents for in vivo molecular profiling of disseminated prostate cancer: Cellular processing of [111 In]-labeled CHX-A"DTPA-trastuzumab and anti-HER2 ABY-025 Affibody in prostate cancer cell lines. Exp Ther Med 2: 523-528, 2011.

16. Andersson $\mathrm{K}$ and Malmqvist M: Method for the analysis of solid biological objects. Patent application WO 2,010,033,069, 2008.

17. Svitel J, Balbo A, Mariuzza RA, Gonzales NR and Schuck P: Combined affinity and rate constant distributions of ligand populations from experimental surface binding kinetics and equilibria. Biophys J 84: 4062-4077, 2003.

18. Barta $\mathrm{P}, \mathrm{Björkelund} \mathrm{H}$ and Andersson $\mathrm{K}$ : Circumventing the requirement of binding saturation for receptor quantification using interaction kinetic extrapolation. Nucl Med Commun 32: 863-867, 2011.

19. Malmberg J, Tolmachev V and Orlova A: Imaging agents for in vivo molecular profiling of disseminated prostate cancer targeting EGFR receptors in prostate cancer: comparison of cellular processing of [ $\left.{ }^{111} \mathrm{In}\right]$-labeled affibody molecule $\mathrm{Z}_{\mathrm{EGFR}: 2377}$ and cetuximab. Int J Oncol 38: 1137-1143, 2011. 